\title{
Impact Cratering: The Effect of Crustal Strength and Planetary Gravity
}

\author{
John D. O'Keefe and Thomas J. Ahrens
}

Seismological Laboratory, California Institute of Technology, Pasadena, California 91125

\begin{abstract}
Upon impact of a meteorite with a planetary surface the resulting shock wave both 'processes' the material in the vicinity of the impact and sets a larger volume of material than was subjected to high pressure into motion. Most of the volume which is excavated by the impact leaves the crater after the shock wave has decayed. The kinetic energy which has been deposited in the planetary surface is converted into reversible and irreversible work, carried out against the planetary gravity field and against the strength of the impacted material, respectively. By using the results of compressible flow calculations prescribing the initial stages of the impact interaction (obtained with finite difference techniques) the final stages of cratering flow along the symmetry axis are described, using the incompressible flow formalism proposed by Maxwell. The fundamental assumption in this description is that the amplitude of the particle velocity field decreases with time as kinetic energy is converted into heat and gravitational potential energy. At a given time in a spherical coordinate system the radial velocity is proportional to $R^{-z}$, where $R$ is the radius (normalized by projectile velocity) and $z$ is a constant shape factor for the duration of flow and a weak function of angle. The azimuthal velocity, as well as the streamlines, is prescribed by the incompressibility condition. The final crater depth (for fixed strength $Y$ ) is found to be proportional to $R_{0}\left[2(z+1) u_{o r}{ }^{2} / g\right]^{1 /(z+1)}$, where $u_{o r}$ is the initial radial particle velocity at (projectile normalized) radius $R_{0,} g$ is planetary gravity, and $z$ (which varied from 2 to 3 ) is the shape factor. The final crater depth (for fixed gravity) is also found to be proportional to $\left[\rho u_{o r}{ }^{2} / Y_{z}\right]^{1 /(z+1)}$, where $\rho$ and $Y$ are planetary density and yield strength, respectively. By using a Mohr-Coulomb yield criterion the effect of varying strength on transient crater depth and on crater formation time in the gravity field of the moon is investigated for $5-\mathrm{km} / \mathrm{s}$ impactors with radii in the $10-$ to $10^{7}-\mathrm{cm}$ range. Comparison of crater formation time and maximum transient crater depth as a function of gravity yields dependencies proportional to $\mathrm{g}^{-0.58}$ and $\mathrm{g}^{-0.19}$, respectively, compared to $\mathrm{g}^{-0.618}$ and $\mathrm{g}^{-0.165}$ observed by Gault and Wedekind for hypervelocity impact craters in the 16- to 26-cm-diameter range in a quartz sand (with Mohr-Coulomb type behavior) carried out over an effective gravity range of $72-980 \mathrm{~cm} / \mathrm{s}^{2}$.
\end{abstract}

\section{INTRODUCTION}

In order to obtain the relative ages of cratered surfaces formed on different terranes on a single planet [e.g., Neukum, 1977], as well as to obtain meaningful interplanetary comparisons [e.g., Gault et al., 1975; Neukum and Wise, 1976], the effect of planetary crustal strength and gravity on the dimensions of craters must be understood. Because the largest manmade impact craters (produced on the moon by empty rocket casings) are only some $40 \mathrm{~m}$ in diameter [Whitaker, 1972] and the largest nuclear explosions (formed in wet coral sand) are only $1.8 \mathrm{~km}$ in diameter [Vaile, 1961], the experimental data available are limited, and theoretical estimates must be used to understand crater populations.

There have been a number of studies entailing the analytical and numerical prediction of cratering phenomena and the scaling of laboratory experimental data to planetary cratering. In order to test theories [e.g., Chabai, 1965] of scaling of the sizes of explosive (and impact) craters in a variety of gravitational fields, centimeter scale explosion cratering experiments have been carried out under conditions both less than the earth's gravity field [Johnson et al., 1969; Gault and Wedekind, 1977] and greater than the earth's gravity field [Victorov and Stepenov, 1960; Schmidt and Holsapple, 1980]. The effect of rock or soil strength on laboratory cratering experiments has been addressed by Moore et al. [1964], Gaffney [1979], and Holsapple and Schmidt [1979], and on the basis of extensive numerical calculations the scaling of hypervelocity impact craters is discussed by Dienes and Walsh [1970]. In the latter paper the conditions are described for similitude of the hydrodynamic flows induced by hypervelocity impacts in materials

Copyright $\mathcal{C} 1981$ by the American Geophysical Union. at sizes and time scales for which gravity does not affect the flow. Theoretically based scaling laws for relating final crater dimensions to target and projectile densities and projectile kinetic energy or momentum (or a combination of these), planetary gravity, sound speed, and material strength have been developed on the basis of laboratory and field data [e.g., Chabai, 1965; Gault, 1973; Gault et al., 1975; O'Keefe and Ahrens, 1978].

In the present paper we examine how maximum transient crater depth is related to projectile energy for a range of planetary gravities and crustal strength conditions. Moreover, we examine how the scaling laws themselves depend on gravity and target strength. Although, in principle, such a study could be carried out by using only the finite difference numerical methods previously applied to meteorite impact cratering on the earth and the terrestrial planets [Bjork, 1961; O'Keefe and Ahrens, 1975, 1976, 1977a, b, 1978; Swift, 1977; Bryan et al., 1978], this would be computationally uneconomical. The approach taken is (1) to establish the validity for impact cratering of the Maxwell $z$ model, which was previously applied to explosive cratering by Maxwell $[1973,1977]$ and Maxwell and Seifert [1976], (2) to compute the partitioning of impact energy into gravitational potential energy for large-scale cratering and the effect of weakening of planetary material via 'shock processing,' and (3) to make a comparison of calculated and laboratory crater excavation times and depths. The physical basis for the incompressible flow description of late stage cratering processes is described in detail in the next section. Specifically, we use the incompressible formalism to examine the effects of planetary crustal strength and gravity on the moon and to compare calculational results with laboratory cratering experiments. 


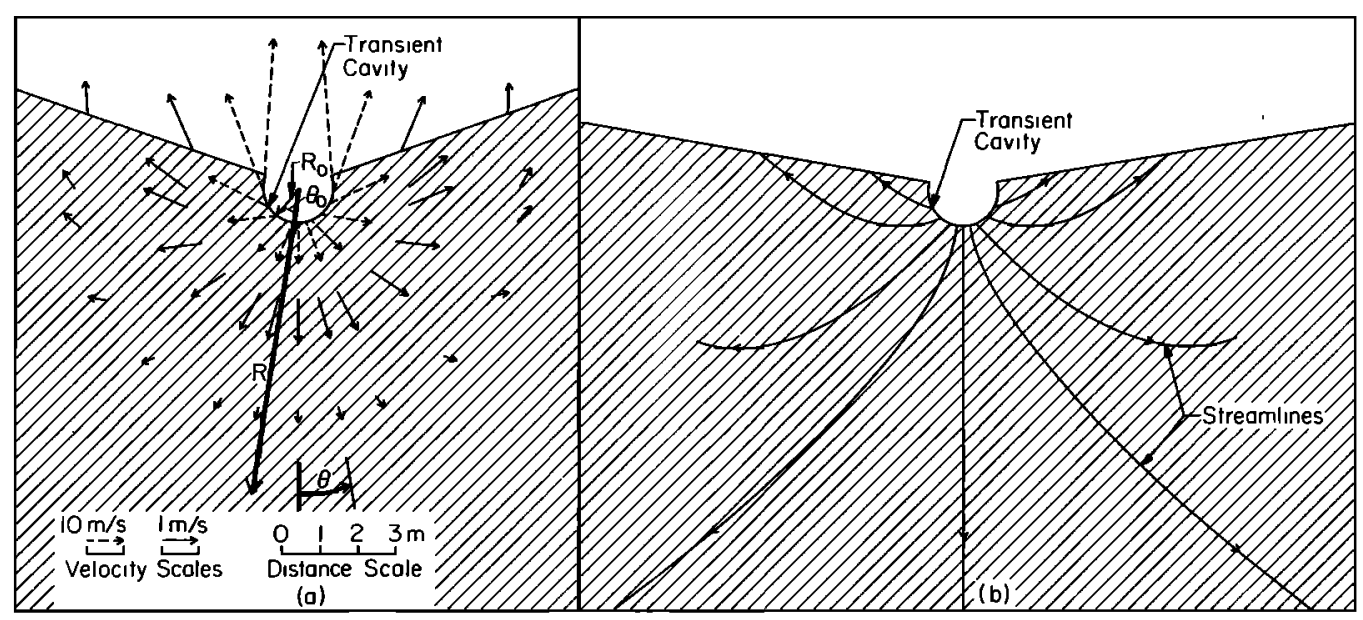

Fig. 1. Incompressible flow field cratering model. (a) Particle velocity vectors for $R_{0}=1 \mathrm{~m}, \alpha=10 \mathrm{~m} / \mathrm{s}$, and $z=3$ for $\theta$ $=0^{\circ}-110^{\circ}$. (b) Streamlines for incompressible flow for $R_{0}=1 \mathrm{~m}, \alpha=10 \mathrm{~m} / \mathrm{s}$, and $z=2$.

\section{Late Stage Cratering Flow}

\section{a. The z Model}

Bjork et al. [1967] first pointed out that the impact of a meteorite with a solid half space, such as a planetary surface, sets a large region of the target into motion after the shock wave has 'processed' a portion of target (planetary material) in the immediate vicinity of the impact and that this large region is much greater in size than that which experienced peak dynamic pressures. The decaying shock wave propagates away from the immediate region of impact and becomes 'detached' from the impactor. Thus a large low-stress region which was set into motion by the shock wave becomes involved in crater excavation. Once the initial impact-induced particle velocity flow in the target has been established and the stresses have decayed such that the kinetic energy per unit mass is much greater than recoverable strain energy, the size of the crater produced is solely controlled by conservation of energy for a fixed geometry, as was suggested in an early model of cratering by Charters and Summers [1959]. We assume in the incompressible late stage flow $z$ model that the kinetic energy of the mass of target material along a given flow streamline is equal to the irreversible work that is performed against the material due to its finite strength plus the work done against the planetary gravity field. Since the total kinetic energy imparted to the impacted target region is bounded as a result of the initial shock and rarefaction wave interaction, the equality of initial kinetic energy along a streamline with the irreversible work done against the material strength and the planetary gravity field limits the final crater dimensions.

The key to utilizing the conservation of energy concept described qualitatively above is that although the shock-induced particle velocities in the flow field decrease in magnitude with time, the geometry of the streamlines remains nearly constant for the duration of the crater excavation process for a given explosion [Maxwell, 1973] and, as we will see in the next section, also for a given impact. The simple assumption made by Maxwell [1973] and demonstrated in section 3, which yields an approximate description of the late stage impact-induced flow, is that for the spherical geometry defined in Figure $1 a$ the radial particle velocity $u_{r}$ at a given time $t$ is given by

$$
u_{r}=d R / d t=\alpha(t) / R^{z}
$$

where $R$ is the projectile-normalized radius, $\alpha(t)$ is a parameter which depends on the strength of the flow, and $z$ is a constant for a given streamline which specifies the shape of the streamline. The validity of the latter statement can be demonstrated by considering the divergence of the flow (equal to zero on account of the incompressibility assumption) in twodimensional spherical coordinates:

$$
0=\sin \theta \partial\left(R^{2} u_{r}\right) / \partial R+R \partial\left(\sin \theta u_{\theta}\right) / \partial \theta
$$

where $\theta$ and $u_{\theta}$ are defined in Figure la. Substituting (1) into (2) and integrating over the angular range $0-\theta$ of the flow

$$
(z-2) u_{r} \int_{0}^{\theta} \sin \theta d \theta=\int_{0}^{\theta} d\left(\sin \theta u_{\theta}\right)
$$

yields, for the azimuthal particle velocity,

$$
u_{\theta}=R d \theta / d t=\sin \theta(z-2) u_{r} /(1+\cos \theta)
$$

Again substituting $d R / d t$ for $u_{r}$ in (4) and integrating from $\theta_{0}$ to $\theta$ and $R_{0}$ to $R$, the trajectory of a streamline (Figure $1 b$ ) is given by

$$
\left(R / R_{0}\right)^{(z-2)}=(1-\cos \theta) /\left(1-\cos \theta_{0}\right)
$$

As is shown in Figure $1 a, R_{0}$ and $\theta_{0}$ refer to a point on a reference surface which is taken to be on the boundary of the crater transient cavity at a given reference time. The time dependence of the particle radial position can be obtained by integrating (1) to yield

$$
R(t)^{z+1}-R_{0}\left(t_{0}\right)^{z+1}=(z+1) \int_{0}^{t} \alpha(t) d t \equiv(z+1)\langle\alpha\rangle t
$$

where $\langle\alpha\rangle$ is the mean value.

b. Transient and Final Cavity

If the crater depth is denoted by $R_{c}$, it follows from (6) that

$$
R_{c}(t) \cong[(z+1)\langle\alpha\rangle t]^{1 /(z+1)}
$$

for $\boldsymbol{R}_{0} \gg R_{c}$. The final crater depth, and hence total crater excavation time, is specified by total energy consideration, for example, (49). Equations (6) and (7) yield a general expression 
for the radial position of a particle $R(l)$ in terms of its initial radial position and the current transient crater depth:

$$
R(t) \cong\left[R_{0}\left(t_{0}\right)^{z+1}+R_{c}(t)^{z+1}\right]^{1 /(z+1)}
$$

which may be expressed as [Maxwell, 1973]

$$
\ln \left[R(t) / R_{0}\right] \cong-\ln \left[1-\left(R_{c} / R_{0}\right)^{(z+1)}\right] /(z+1)
$$

\section{c. Volume Integrals}

In order to compute (1) the loss in kinetic energy as a result of energy dissipation due to finite strength and (2) work done against the gravitational field (as is described in section 3 ) we require an expression for a volume element along a stream tube. A stream tube of initial area $A_{0}$ at reference radius $R_{0}$ with velocity $u_{0}$ is shown with an area $A$ at a radius $R$ in Figure 2 . The incompressible assumption implies that

$$
A u=A_{0} u_{0}
$$

and

$$
u=\left(u_{r}^{2}+u_{\theta}^{2}\right)^{1 / 2}
$$

which from (1) and (4) is given as

$$
u=\alpha\left\{1+[\sin \theta(z-2) /(1+\cos \theta)]^{2}\right\}^{1 / 2} / R^{2}
$$

Denoting values of $A, u, R$, and $\theta$ at the reference surface with the subscript zero, and substituting (11) and (12) into (10) yields

$$
\begin{array}{r}
A=A_{0}\left(R / R_{0}\right)^{2}\left\{1+\left[\sin \theta_{0}(z-2) /\left(1+\cos \theta_{0}\right)\right]^{2}\right\}^{1 / 2} / \\
\left\{1+[\sin \theta(z-2) /(1+\cos \theta)]^{2}\right\}^{1 / 2}
\end{array}
$$

since a volume element along a streamline is given by

$$
d V=A d S
$$

where $d S$ is the differential along the streamline given by

$$
d S=d R / \cos \psi=u d R / u_{r}
$$

and $\psi$ is defined in Figure 2.

Substituting (12) and (13) into (15) and this expression into (14) yields, for the volume element along a streamline,

$$
d V=A_{0}\left(R / R_{0}\right)^{2}\left\{1+\left[\sin \theta_{0}(z+2) /\left(1+\cos \theta_{0}\right)\right]^{2}\right\}^{1 / 2} d R
$$

\section{d. Strain Energy}

Whereas the decrease in kinetic energy and gain in gravitational energy for a volume element may be simply expressed, the strain energy rate must be given in terms of the principal

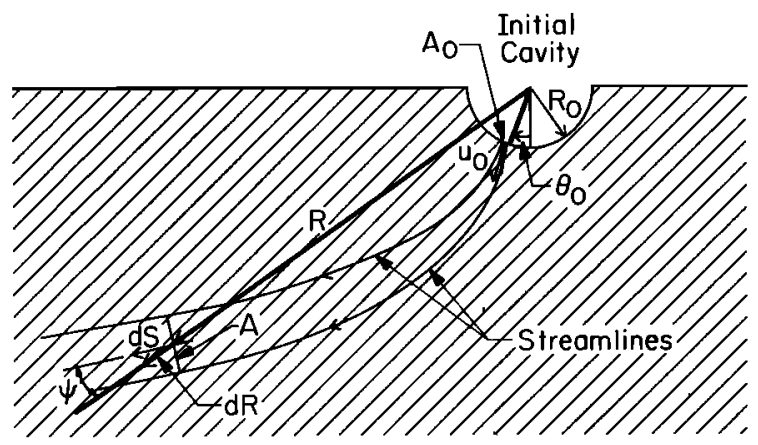

Fig. 2. Relation of surface element $A$ to line element $d S$ and streamlines.

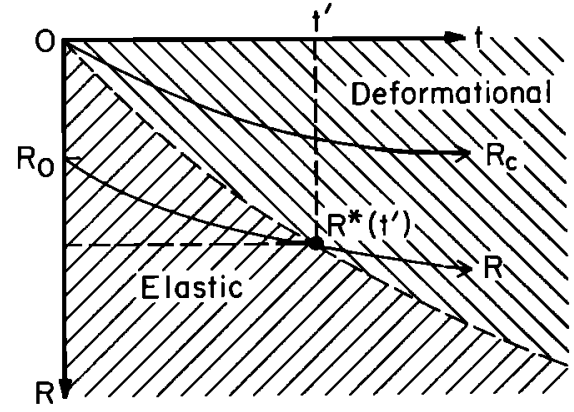

Fig. 3. Radius versus time diagram showing the trajectory of a general point along a radius $R$, the crater depth $R_{c}$, and the elastic deformational boundary versus time.

radial $\left(d \epsilon_{r} / d t\right)$, angular $\left(d \epsilon_{\theta} / d t\right)$, and azimuthal $\left(d \epsilon_{\phi} / d t\right)$ strain rates [Batchelor, 1967, p. 601]:

$$
\begin{aligned}
& d \epsilon_{r} / d t=\partial u_{r} / \partial R=-z(d R / d t) / R=-\alpha z / R^{(z+1)} \\
& d \epsilon_{\theta} / d t=\left(\partial u_{\theta} / \partial \theta\right) / R+u_{r} / R=u_{r}[1+(z-2) /(1+\cos \theta)] / R
\end{aligned}
$$

$d \epsilon_{\phi} / d t=\left(\partial u_{\phi} / \partial \phi\right) /(R \sin \theta)+u_{r} / R+u_{\theta} \cot \theta / R$

$$
=\alpha[1+(z+2) \cos \theta /(1+\cos \theta)] / R^{(z+1)}
$$

where from cylindrical symmetry, $\partial u_{\phi} / \partial \phi=0$.

We note, following Maxwell [1973], that for the on-axis strain rate $(\theta=0)$,

$$
d \epsilon_{\theta} / d t=d \epsilon_{\phi} / d t=-\left(d \epsilon_{r} / d t\right) / 2
$$

and the principal stress difference between the radial stress $p_{\text {r }}$ and azimuthal stress $p_{\theta}$ is less than or equal to the Tresca yield stress $Y$ in the spherical approximation:

$$
\left|p_{r}-p_{\theta}\right| \leq Y
$$

From (20) and Hooke's law it follows that

$$
2 \mu\left[\epsilon_{r}-\left(-\epsilon_{r} / 2\right)\right] \leq-Y
$$

or

$$
\left.3 \mu \epsilon_{r}\right|_{R \leq R^{*}}=-Y
$$

at the position where $R \leq R^{*}$. Here $\epsilon_{r}$ is the maximum elastic radial strain and $R^{*}$ is the point beneath the crater where transition occurs between material which has and has not yielded (Figure 3).

Denoting $\left.\epsilon_{r}\right|_{R \leq R^{*}}$ from (17) as

$$
\epsilon_{r}=-z \int_{R_{0}}^{R} d R / R=-z \ln \left(R / R_{0}\right)
$$

yields, upon substitution from (9),

$$
\epsilon_{r} \cong z \ln \left[1-\left(R_{c} / R_{0}\right)^{(z+1)}\right] /(z+1)
$$

which upon substitution into (22) yields

$$
-Y(z+1) /(3 \mu z) \cong \ln \left[1-\left(R_{c} / R_{0}\right)^{(z+1)}\right]
$$

which specifies the radius to the elastic behavior surface in terms of the radius to the interface between the yielded and not yet yielded material versus crater depth.

Since, in general, $|Y| / \mu \ll 1$, (25) may be simplified to

$$
\left(R_{c} / R^{*}\right)^{(z+1)} \cong Y(z+1) / 3 \mu z
$$


For an elastic incompressible medium the (shear) strain energy per unit volume is given by

$$
\begin{array}{r}
\eta_{e}=\mu \int_{0}^{t}\left(\epsilon_{r} d \epsilon_{r} / d t+\epsilon_{\theta} d \epsilon_{\theta} / d t+\epsilon_{\phi} d \epsilon_{\phi} / d t\right) d t \\
\eta_{e}=\mu \int_{0}^{t}\left\{(-z) \ln \left(R / R_{0}\right)(-z) d(\ln R) / d t+2[(z / 2)\right. \\
\left.\cdot \ln \left(R / R_{0}\right)(z / 2) d \ln \left(R / R_{0}\right) / d t\right\} d t \\
\eta_{e}=1.5 \mu z^{2} \ln ^{2}\left(R / R_{0}\right)
\end{array}
$$

Using (9) gives

$$
\eta_{e}=1.5 \mu[z /(z+1)]^{2} \ln ^{2}\left[1-\left(R_{c} / R_{0}\right)^{(z+1)}\right]
$$

or using (22) and (23) in (29) yields

$$
\eta_{e}=Y^{2} / 6 \mu
$$

In the case of material which has yielded, the energy density deposited by work carried out on the medium is given by the sum of the elastic work plus the plastic work. The latter is given by

$$
\eta_{d}=-Y \int_{r^{\prime}}^{t} \epsilon_{r} d t
$$

where $t^{\prime}$ is the time required for the stress at a depth $R^{*}$ to exceed the material yield strength $-Y$. Substituting (23) into (32) yields

$$
\eta_{d} \cong Y z \ln \left(R / R^{*}\right)=Y z\left[\ln \left(R_{0} / R^{*}\right)+\ln \left(R / R_{0}\right)\right]
$$

Inverting the argument of the first term in (33) and substituting (9) for the second term yields

$$
\eta_{d} \cong-Y_{z} \ln \left(R^{*} / R_{0}\right)-Y_{z} \ln \left[1-\left(R_{c} / R_{0}\right)^{(z+1)}\right] /(z+1)
$$

Using (23) and then (22) to substitute in the first term of (34) yields

$$
\eta_{d} \cong Y^{2} / 3 \mu-Y z \ln \left[1-\left(R_{c} / R\right)^{(z+1)}\right] /(z+1)
$$

In the elastic-plastic deformation region the total strain energy is given by

$$
\eta=\eta_{e}+\eta_{d}
$$

\section{e. Energy Integrals}

Some simple equations for final crater depth versus planetary gravity and (constant) strength in terms of initial crater parameters may be obtained by considering integrals of the form

$$
\begin{aligned}
& E_{e}=\int_{R^{*}}^{\infty} \eta_{e} d V \\
& E_{d}=\int_{R_{c}}^{R^{*}} \eta_{d} d V
\end{aligned}
$$

where the volume element for the axial stream tube from (16) for $\theta=0$ is

$$
d V=A_{0}\left(R / R_{0}\right)^{2} d R
$$

Substituting (30) and (39) into (37) yields

$$
E_{e} \cong 1.5 \mu A_{0}[z /(z+1)]^{2} / R_{0} z \int_{R^{*}}^{\infty} \ln ^{2}\left[1-\left(R_{c} / R_{0}\right)^{(z+1)}\right] R^{z} d R
$$

which may be approximated by

$$
E_{e} \cong 1.5 \mu A_{0}[z /(z+1)]^{2} R_{c}^{2(z+1)} / R_{0}^{z} \int_{R^{*}}^{\infty} R^{-(z+2)} d R
$$

Integrating (41) and using the approximation of (26) yields

$$
E_{e} \cong 0.5 Y z A_{0} R_{c}^{(z+1)} /\left[(z+1)^{2} R_{0}^{z}\right]
$$

A more complete expression for the energy in the axial stream tube of an elastic-plastic target may be obtained by substituting into the sum of integrals of the forms of (37) and (38) using (31) and (35). This yields

$$
\begin{aligned}
E_{\tau^{*}}=E_{e}+E_{d} \cong & Y^{2} A_{0} /\left[2 \mu R_{0}{ }^{z}\right] \int_{R_{c}}^{R^{*}} R^{z} d R^{\prime} \\
& +Y A_{0} z R_{c}^{(z+1)} /\left[R_{0}^{z}(z+1)\right] \int_{R_{c}}^{R^{*}} d R / R
\end{aligned}
$$

where the second term in (35) has been simplified by the approximation of (26). Using (26) to simplify the results of both integrals in (43) yields

$$
\begin{aligned}
E_{s} \cong A_{0} Y R_{c}^{(z+1)} /(z+1) & R_{0}^{2}\{1.5 z-Y / 2 \mu \\
+ & z \ln [3 \mu z / Y(z+1)] /(z+1)\}
\end{aligned}
$$

The gravitational potential energy $E_{g}$ involved in excavating the axial stream tube of area $A_{0}$ in a planetary surface of density $\rho$ is

$$
E_{g}=A_{0} \rho \int_{0}^{R_{c}} g R^{2+1} d R
$$

or, if $g$ is assumed to be nearly independent of $R$,

$$
E_{g}=A_{0} \rho g R_{c}^{(2+2)} /(z+2)
$$

Equations (42) and (46) were also given by Maxwell [1973]. Finally, the initial kinetic energy in the axial stream tube at $t$ $=t_{0}$ is given, using ( 1 ), by

$E_{K E}=\left[A_{0} \rho / 2\right] \int_{R_{0}}^{\infty}\left[\alpha\left(t_{0}\right) / R^{z}\right]^{2} R^{z} d R=-A_{0} \rho \alpha^{2}\left(t_{0}\right) R_{0}{ }^{(1-z)} /(2 z-2)$

In order to observe the effect of varying $R_{0}, g$, and $Y$ on the maximum transient depth crater it is useful to substitute the value of the initial particle velocity $u_{o r}$ in (47) to yield

$$
E_{K E}=-\rho u_{o r}^{2} R_{0}^{(z+1)} /(2 z-2)
$$

Equating the initial kinetic energy in the axial tube to the strain energy generated by producing the crater, the latter, either specified by (42) or (44) plus the gravitational energy of excavation of the axial tube, yields, for the final to initial crater depth ratio,

$R_{c} / R_{0} \cong\left(\rho u_{o r}^{2}\right)^{1 /(z+1)} /\left(2(z+1)\left[\rho g /(z+1)+0.5 Y z / R_{0}^{2}\right]\right]^{1 /(z+1)}$

If $Y=0$, we obtain

$$
R_{c} / R_{0} \cong\left[2(z+1) u_{o r}^{2} / g\right]^{1 /(z+1)} / 2(z+1)
$$

whereas when $g=0$,

$$
R_{c}=\left(2 \rho u_{o r}{ }^{2} / Y z\right)^{1 /(z+1)} / 2(z+1)
$$

Using the results of section 3 , in which values of $z$ between 2 and 3 are obtained for the centerline streamline, (50) and 
(51a) are useful to predict the effect on maximum excavation depth of changing planetary gravity and strength for differently shaped cratering flows. Maxwell [1977] shows that for craters for which $z=$ const, for the entire incompressible flow regime,

$$
R_{c} \propto g^{-1 /(2 z+1)}
$$

We demonstrate in Figure 6, and more recently, Austin et al. [1980] have also shown, that for impact-induced flows, $z$ increases from a value between 2 and 3 along the centerline of the flow to values of $\sim 3.5-4$ near $\theta=90^{\circ}$.

\section{f. Variable Yield Strength}

In (31) and (32) we have treated $-Y$ as a constant in the integration over time. Whereas on a laboratory scale such an assumption may be adequate only if the coefficient of internal friction is zero, on a planetary scale, if it is assumed that crustal strength increases with overburden pressure or mean principal stress as

$$
\mid \boldsymbol{Y}=Y_{1}+a \bar{P}
$$

where $\bar{P}$ is mean principal stress or pressure $P$ and $|Y|$ is allowed to increase until a maximum value $Y_{3}$ is achieved (the von Mises limit), a more complicated model should be considered. In addition to this static contribution to the pressure, although the late stage flow is incompressible, the vorticity of the flow gives rise to a dynamic pressure which will increase the effective pressure (D. E. Maxwell, private communication, 1979). In order to describe the dynamic pressure $P$ along an axial stream tube we consider one-dimensional flow along $\boldsymbol{R}$ with variable area $A$. Conservation of momentum and mass is then

$$
\begin{gathered}
d u_{r} / d t=\partial u_{r} / \partial t+u_{r} \partial u_{r} / \partial R=-\left(\partial \sigma_{r} / \partial R\right) / \rho \\
A \partial u_{r} / \partial R+u_{r} \partial A / \partial R=0
\end{gathered}
$$

hence

$$
\partial u_{r} / \partial R=-u_{f} f
$$

where

$$
f \equiv(\partial A / \partial R) / A
$$

Operating on (53) with $\partial / \partial R$ yields

$\partial^{2} u_{r} / \partial R \partial t+u_{r}\left(\partial^{2} u_{r} / \partial R^{2}\right)+\left(\partial u_{r} / \partial R\right)^{2}=-\left(\partial^{2} \sigma_{r} / d R^{2}\right) / \rho$

which, using (55), may be written as

$$
-f(d u / d t)+u^{2}\left(f^{2}-\partial f / \partial R\right)=-\left(\partial^{2} \sigma_{r} / \partial R^{2}\right) / \rho
$$

TABLE 1. Radial Stresses $\sigma_{r}$ Derived From Velocity Gradients, Lunar Gravity, and Vorticity for $z$ Model Flow

\begin{tabular}{clll}
\hline $\begin{array}{c}\text { Radius, } \\
\text { cm }\end{array}$ & $\begin{array}{c}\text { Velocity } \\
\text { Pressure, } \\
\text { Mbar }\end{array}$ & $\begin{array}{c}\text { Lithostatic } \\
\text { Stress, } \\
\text { Mbar }\end{array}$ & $\begin{array}{c}\text { Vorticity } \\
\text { Stress, } \\
\text { Mbar }\end{array}$ \\
\hline $9.19 \dagger$ & 0 & 0 & 0 \\
28 & $8.1 \times 10^{-4}$ & $1.2 \times 10^{-8}$ & $1.5 \times 10^{-5}$ \\
66 & $3.4 \times 10^{-4}$ & $2.5 \times 10^{-8}$ & $4.4 \times 10^{-5}$ \\
142 & $1.02 \times 10^{-4}$ & $4.3 \times 10^{-8}$ & $2.0 \times 10^{-8}$ \\
180 & $2.7 \times 10^{-5}$ & $5.5 \times 10^{-8}$ & $4.9 \times 10^{-9}$ \\
\hline
\end{tabular}

*From the lunar surface $\left(2.94 \mathrm{~g} / \mathrm{cm}^{3}\right), Y_{1}=Y_{3}=10^{-7} \mathrm{Mbar}$ impacted by a $10-\mathrm{cm}$-diameter projectile.

$t$ The radius of the inner boundary at $t=0$ was $9 \mathrm{~cm}$. After $2.57 \mu \mathrm{s}$ of flow the boundary moved $0.19 \mathrm{~cm}$ downward.
Substituting for $d u / d t$ from (53) yields

$$
\left(\partial \sigma_{r} / \partial R\right) f+\partial^{2} \sigma_{r} / \partial R^{2}=\rho u_{r}^{2}\left(f^{\prime}-f^{2}\right)
$$

where $f^{\prime} \equiv \partial f / \partial R$.

From (13), for $\theta=0$ we infer that

$$
A=A_{0}^{\prime} R^{z}
$$

where

$$
A_{0}^{\prime}=A_{0} / R_{0}^{z}
$$

then

$$
\begin{gathered}
f=z / R \\
f^{\prime}=-z / R^{2}
\end{gathered}
$$

Substituting (62) and (63) with (59) yields

$$
\partial^{2} \sigma_{r} / \partial R^{2}+\left(\partial \sigma_{r} / \partial R\right) z / R=-\rho[\alpha(t)]^{2} z(z+1) / R^{(2 z+2)}
$$

Assuming a general solution of (64) of the form

$$
\sigma_{r}=[\alpha(t)]^{2} \Omega(R)
$$

yields upon substitution

$$
\Omega^{\prime \prime}+z \Omega^{\prime} / R=-\rho z(z+1) / \sigma_{r}^{2 z+2}
$$

Assuming a form for $\Omega$ such that

$$
\Omega=G \boldsymbol{R}^{m}
$$

yields, upon substitution and equating, exponents and coefficients of $R$

$$
\begin{gathered}
m=-2 z \\
G=-\rho / 2
\end{gathered}
$$

respectively.

The homogeneous form of (66),

$$
\Omega^{\prime \prime}+z \Omega^{\prime} / R=0
$$

has a solution of the form

$$
\Omega=A_{1} R^{(1-z)}+A_{2}
$$

Adding the solutions of the particular and homogeneous equation and taking into account (65) yields

$$
\sigma_{r}=\left[A_{1} R^{(1-z)}+A_{2}-\rho R^{-2 z} / 2\right][\alpha(t)]^{2}
$$

Applying the boundary conditions that at $R \rightarrow \infty, \sigma_{r}=0$, and at $R=R_{c}, \sigma_{r}=0$ yields

$$
\begin{gathered}
A_{2}=0 \\
A_{1}=\rho /\left[2 R_{c}^{(2+1)}\right]
\end{gathered}
$$

respectively; thus the final solution of $(64)$ is

$$
\sigma_{r}=[\alpha(t)]^{2}\left\{R^{(1-z)} / R_{c}^{(1+z)}-R^{-2 z}\right\} / 2
$$

In calculating the pressure from the increased dynamic radial stress to specify the yield strength along the axis stream tube according to (52) the pressure calculated from (75) must be added to the lithostatic pressure.

In the initial stages of incompressible flow, when the velocity gradient is high, $\sigma_{r}$ as calculated from (75) dominates the principal stresses. As is demonstrated in Table 1, $\sigma_{r}$ varied from 1 to $10^{4}$ times either the gravitationally induced stress or that produced by the vorticity of the flow. 

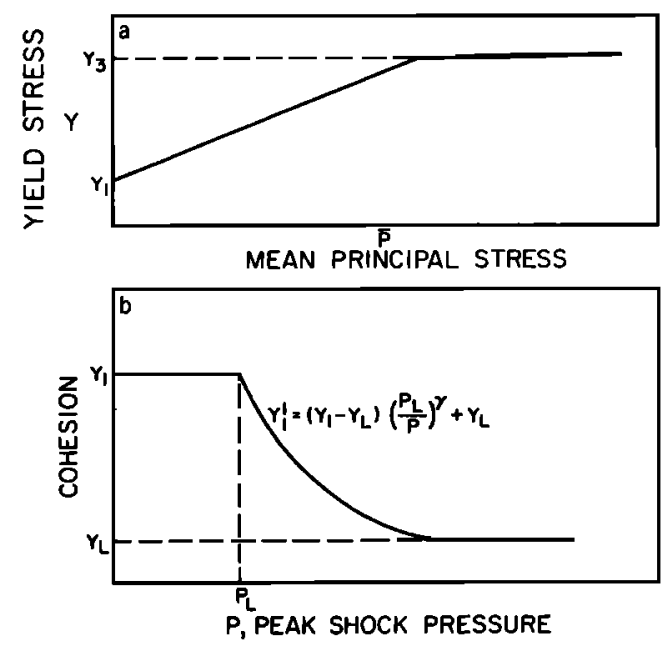

Fig. 4. Strength models used in incompressible flow calculations. (a) Mohr-Coulomb model; $Y_{1}$ represents cohesive strength, and $Y_{3}$ represents maximum or von Mises strength. (b) Shock-induced strength degradation model described by (76).

\section{Material Strength Models}

The general material strength model, used in this study, is a Mohr-Coulomb type with a von Mises limit, as already discussed in relation to (52). Referring to Figure $4 a$, the material under zero mean stress has a cohesion $Y_{1}$, which increases linearly with pressure as given by (51a), where $a$, the coefficient of internal friction, is a material constant. The value of the yield stress is limited to values less than or equal to the von Mises limit $\boldsymbol{Y}_{\mathbf{3}}$.

A number of researchers, including Knowles and Brode [1977], Orphal [1977], and Swift [1977], have proposed that the passage of the initial shock wave through rock, preceding crater excavation, weakens the rock. Swift [1977] carried out a series of explosive excavation calculations with a shock-induced degradation of the material strength model.

The model used for shock degradation of material strength is shown in Figure $4 b$. Referring to Figure $4 b$, if the peak shock stress is less than a minimum stress for failure, $P_{L}$, then there is no change. If the peak stress is greater than $P_{L}$, then the cohesion is given by

$$
Y_{1}^{\prime}=\left(Y_{1}-Y_{L}\right)\left(P_{L} / P\right)^{\gamma}+Y_{L}
$$

where $Y_{L}$ is a lower limit on the shock alteration of the cohesion.

\section{Relating InCOMpressible Flow (z) MOdels to Compressible Flow Calculations}

As was discussed in section $2 e$, the incompressible ( $z$ ) model requires initial values for the parameters $\alpha\left(t_{0}\right)$ and $z$ at $R_{0}\left(t_{0}\right)$. These have been obtained from compressible flow calculations but could, in principle, also be derived from experi-
TABLE 3. Material Strength Model Used in Compressible Flow Calculations

\begin{tabular}{lc}
\hline \hline \multicolumn{1}{c}{ Equation } & Condition \\
\hline$Y=\left(A+B \mu+C \mu^{2}\right)\left(1-E / E_{m}\right)$ & $E \leq E_{m}$ \\
$Y=0$ & $E>E_{m}$ \\
\hline Parameter & Value \\
\hline$A$, kbar & 2.69 \\
$B$, kbar & 33.8 \\
$C$, kbar & -901.8 \\
$E_{m}, 10^{10} \mathrm{ergs} / \mathrm{g}$ & 1.70 \\
\hline \hline
\end{tabular}

$A, B$, and $C$ are from $O$ 'Keefe and $A$ hrens [1976]. $E_{m}$ is the internal energy for incipient melting under standard conditions, and $E$ is internal energy.

mental measurements. We have previously computed the flow fields due to the impact of anorthosite and iron projectiles on an anorthosite half space for impact velocities ranging from 5 to $45 \mathrm{~km} / \mathrm{s}$ [O'Keefe and Ahrens, 1977b]. The material strength model was based on Hugoniot elastic limit measurements by Ahrens et al. [1973] and is representative of strong rocks. In addition to these finite strength calculations we have, by using the formalism described by $O^{\prime}$ Keefe and Ahrens [1977b] and Ahrens and $O$ 'Keefe [1977], computed the flow fields for anorthosite projectiles impacting an anorthosite half space at velocities from 5 to $45 \mathrm{~km} / \mathrm{s}$ under the assumption of zero target and projectile strength. The equation of state and material strength parameters used are listed in Tables 2 and 3. In Figure 5 we examine both the zero and the nonzero strength 5$\mathrm{km} / \mathrm{s}$ impact of anorthosite upon anorthosite to determine the applicability of the incompressible flow equations outlined in section 2.

The applicability of (1) was determined by plotting the radial velocity field averaged over a given angular increment at various times in the crater evolution for the $5-\mathrm{km} / \mathrm{s}$ case. Referring to Figure 6, we see that the velocity profile behind the subkilobar shock is approximately satisfied by (1). Values shown for the parameters $\alpha$ and $z$ were obtained by fitting the flow region behind the shock wave. Since the shock strength is expected to decrease as $\theta$ increases, the average value of $\alpha$ decreases with increasing angular coverage. The value of $z$ near the axis and averaged over $0^{\circ}-10^{\circ}$ is $2.1 \pm 0.2$, and the value averaged over the whole flow field is $3.0 \pm 0.2$ (see also Austin et al. [1980]). This latter value is approximately equal to the value $z=2.7$ found by Maxwell and Seifert [1976] for calculations of cratering flow from buried nuclear explosions. Moreover, from the work of Oberbeck [1971] it has been shown that the flow fields from shallow buried explosive simulate those found in impact events; thus general agreement would be expected.

The results of the calculations of the evolution of the crater radius are shown in Figure 5. At early times, in the shock wave-dominated regime the crater growth rate is identical for the zero and nonzero strength cases. Thus the transfer of the

TABLE 2. Equation of State Parameters Used in Compressible Flow Calculations

\begin{tabular}{cccccc}
\hline & $\begin{array}{c}\text { Density, } \\
\mathrm{g} / \mathrm{cm}^{3}\end{array}$ & $b$ & $\begin{array}{c}A, \\
\mathrm{Mbar}\end{array}$ & $\begin{array}{c}B, \\
\mathrm{Mbar}\end{array}$ & $\begin{array}{c}E_{0}, \\
10^{12} \mathrm{ergs} / \mathrm{g}\end{array}$ \\
\hline $\begin{array}{c}\text { Gabbroic anorthosite } \\
\begin{array}{c}\text { (low-pressure phase) } \\
\text { Gabbroic anorthosite } \\
\text { (high-pressure phase) }\end{array}\end{array}$ & 2.936 & 0.145 & 0.705 & 0.751 & 4.89 \\
\hline
\end{tabular}

The parameters are from the Tillotson equation of state [Ahrens and O'Keefe, 1977]. 
projectile kinetic energy into the initial target velocity field is insensitive to material strength. At later times the solutions diverge and enter a regime in which the growth rate is approximately constant. Maxwell and Seifert [1976] found that for explosion cratering in porous and nonporous materials the velocity and stress fields behind the initial shock wave were the same. The crater growth rate is not strongly dependent upon strength; however, the duration of this regime is material strength dependent. In the case of strong materials the duration of this regime is short and is dominated by plastic work. In contrast, for weak materials the duration is long and is terminated by gravitational work. It is the latter, or coasting, regime that results in very long computer run times for compressible flow calculations for low-strength geologic materials. The compressible flow code solutions were not carried out to the final crater depth because of this constraint. One of the major advantages of the $z$ model is the efficiency achieved in calculations in the late time regime. The variable yield $z$ model given by (52) and (75) was used to compute the crater depth evolution under the assumption of strength values corresponding to the detailed code calculations (see Table 3). The effect of reducing the cohesive strength was to increase the crater depth by more than a factor of 4 and the crater formation time by more than 2 orders of magnitude.

\section{Partitioning OF ENERGY}

Using the results of a compressible flow calculation of the impact of a $5-\mathrm{km} / \mathrm{s}$ anorthosite sphere into an anorthosite half space depicted in Figures 5 and 6, the partitioning of energy in the axial stream tube was computed by using the values of $\alpha\left(t_{0}\right)$ and $z$ obtained for the $\theta=0^{\circ}-10^{\circ}$ sector. A shock degradation model for material strength was not assumed in these calculations. The $z$ model parameters used are summarized in Table 4. There are several possible forms that the impacting energy may take. The partitioning of energy for impact at various scales (assuming 10- to $\left(2 \times 10^{7}\right)$-cm diameter projectiles) was studied by calculating the total plastic work (equation (35)), elastic energy (equation (31)), and gravitational potential energy (equation (46)) in the axial flow tube for the gravity field of the moon. For each case studied, approximately $10^{2}$ time steps with several iterations per time step were carried out, using the above referenced integrals, to reduce all the initial kinetic energy in the flow tube to elastic, deformational,

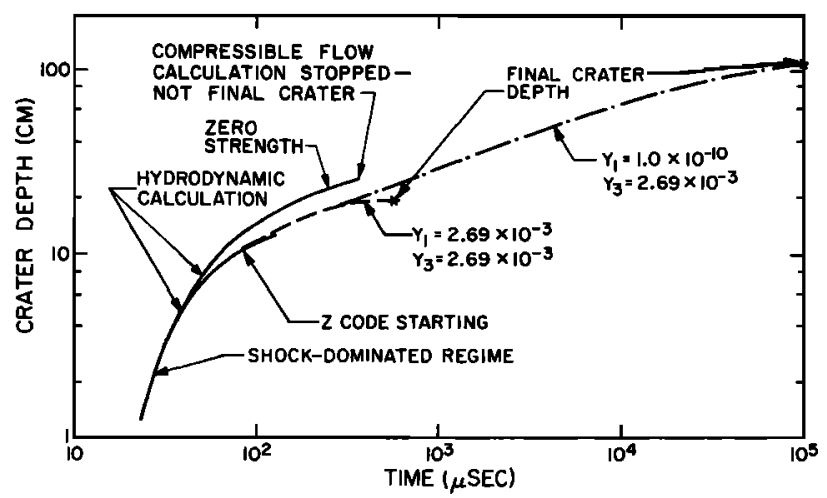

Fig. 5. Crater depth versus time calculated by using compressible flow calculation and linked incompressible calculations ( $z$ code) for the case of zero strength and various Mohr-Coulomb strength crusts with lunar surface gravity. Asterisks indicate final crater depths. Units of yield strength are megabars.

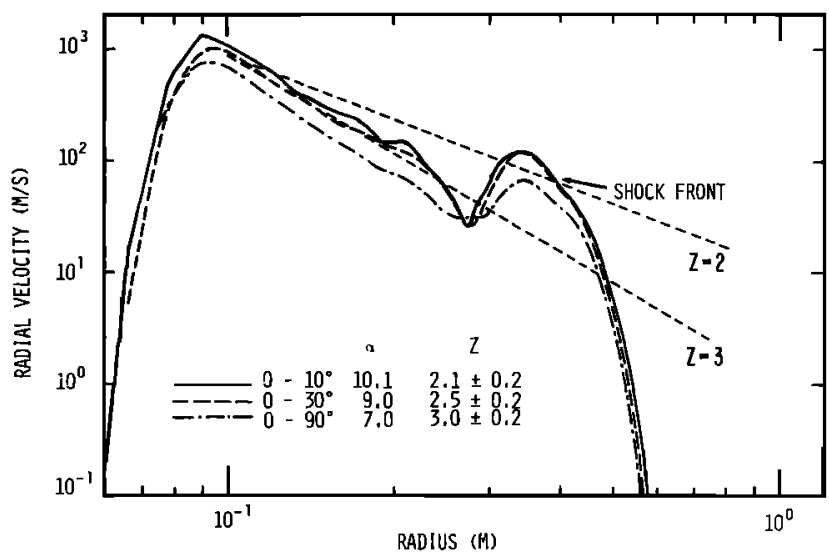

Fig. 6. Calculated radial particle velocity distribution for $5-\mathrm{km} / \mathrm{s}$ impact of anorthosite into anorthosite, $60 \mu \mathrm{s}$ after the impact of a 10 cm-diameter anorthosite sphere, demonstrating average values of a and $z$ which fit (1), upon averaging over different intervals in $\theta$. Units of $\alpha$ are kilometers per second.

and gravitational energy. The initial projectile kinetic energy can be converted irreversibly by shock heating or deformational work into internal energy or stored reversibly as elastic or gravitational energy. Irreversible heating of the axial stream tube material occurs as a result of hydrodynamic shock and is independent of scale for fixed impact velocity and is a constant fraction $(\sim 0.3)$ of the total kinetic energy. This was computed by using the compressible flow formulation described by $O^{\prime} K e e f e$ and Ahrens [1977b]. Plastic work occurs at both high and low stress levels and was computed by using both the compressible and the incompressible formulations. The gravitational energy is important at late times and was computed by using the incompressible method. The partitioning of energy as a function of meteorite radius is shown in Figures $7 a$ and $7 b$ for von Mises and Mohr-Coulomb-von Mises materials, respectively.

Referring to Figure $7 a$, in a von Mises material $\left(Y_{1}=Y_{3}=\right.$ $2.7 \times 10^{-3}$ Mbar) the shock and plastic work account for $75 \%$ of the initial kinetic energy, and the stored elastic energy accounts for $25 \%$ for anorthosite meteorites having radii less than $2 \times 10^{5} \mathrm{~cm}$. For meteorite radii greater than $2 \times 10^{5} \mathrm{~cm}$ the stored elastic energy is decreased, and gravitational potential energy increased, the latter being completely dominant at radii greater than $10^{7} \mathrm{~cm}$.

In the case of Mohr-Coulomb-von Mises materials ( $Y_{1} \leq 1$ $\times 10^{-5}, Y_{3}=2.7 \times 10^{-3} \mathrm{Mbar}$ ) the shock and deformational work account for nearly all of the initial kinetic energy for meteorite radii less than $10^{4} \mathrm{~cm}$. In the range of meteorite radii between $10^{4}$ and $10^{7} \mathrm{~cm}$ the overburden pressure increases the yield stress, and part of the energy is partitioned into stored elastic energy. At meteorite radii greater than $2 \times$ $10^{5} \mathrm{~cm}$ the gravitational energy starts to become important.

TABLE 4. Summary of $z$ Model Parameters Used in Cratering Calculations

\begin{tabular}{ccccl}
\hline & \multicolumn{2}{c}{ Material Strength Parameters } & Typical \\
\cline { 2 - 4 } Case & $Y_{1}$, Mbar & $Y_{2}$, Mbar & $Y_{3}$, Mbar & Medium \\
\hline 1 & $10^{-10}$ & 1.0 & $10^{-10}$ & fluid \\
2 & $10^{-10}$ & 1.0 & $2.7 \times 10^{-3}$ & dry sand \\
3 & $10^{-5}$ & 1.0 & $10^{-5}$ & hard clay \\
4 & $10^{-5}$ & 1.0 & $2.7 \times 10^{-3}$ & soft rock \\
5 & $2.7 \times 10^{-3}$ & 1.0 & $2.7 \times 10^{-3}$ & hard rock \\
\hline
\end{tabular}

The density is $2.94 \mathrm{~g} / \mathrm{cm}^{3}$, and the initial velocity is $0.075 \mathrm{~km} / \mathrm{s}$. 


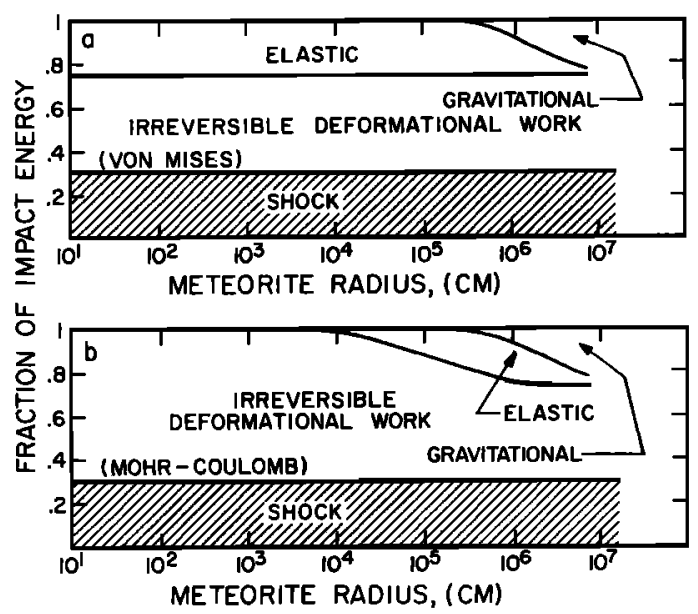

Fig. 7. Energy partitioning versus meteorite radius for a $5-\mathrm{km} / \mathrm{s}$ impact of anorthosite on anorthosite in the gravity field of the moon, where the strength of the planetary surface is assumed (a) to be a constant 2.7 kbar and $(b)$ to increase from a cohesive strength of 10 bars to a maximum strength of $2.7 \mathrm{kbar}$ as a Mohr-Coulomb solid.

\section{Crater formation as a Function OF STRENGTH AND GRAVITY}

Upon calculating crater depth formation times for lunar gravity for a $5-\mathrm{km} / \mathrm{s}$ impact of anorthosite on anorthosite as a function of meteorite radius $R_{m}$ (Figure 8 ) it was discovered that the results are approximately describable in terms of different assumed material strength properties (Table 4). These regimes are (1) cohesive strength, (2) Mohr-Coulomb, (3) von Mises, and (4) gravity. The first three regimes are determined by the material strength. In the cohesive strength regime $\left(\boldsymbol{R}_{m}\right.$ $<10^{4} \mathrm{~cm}$ ) the cohesive strength of the material dominates, and the final crater formation time scales as meteorite radius $\boldsymbol{R}_{m}$. For the moon the range of meteorite radii separating the cohesive strength-dominated crater growth and the MohrCoulomb-dominated growth is around $10^{3} \mathrm{~cm}$ and is dependent upon the specific combination of $Y_{1}$ and $Y_{3}$ assumed. In the Mohr-Coulomb regime $\left(R_{m} \simeq 10^{4}-10^{6}\right)$ the overburden stress increases the yield stress of Mohr-Coulomb materials, and the formation time scales as $\boldsymbol{R}_{m}{ }^{\beta}$, where $0<\beta<1$. In terms of impacting meteorite radius the Mohr-Coulomb regime extends to radii at which the overburden stress increases the yield strength of the bulk of the material involved in the excavation process to the von Mises limit. In the von Mises regime $\left(R_{m} \sim 10^{5} \mathrm{~cm}\right)$ the magnitude of the cohesive strength is less important than the von Mises limit. Here the crater formation time again scales linearly with meteorite radius. Finally, in the gravity regime $\left(R_{m}>\sim 10^{6}-10^{7} \mathrm{~cm}\right)$ the gravitational work of excavation dominates the work done against the material strength. In this regime the crater formation time is proportional to $R_{m}^{1 / 2}$.

The crater depth was also computed as a function of the meteorite radius. As in the case of the crater formation times, the calculational results obtained can be cast in terms of four regimes. In the cohesive regime the crater depth for a given radius can vary by over an order of magnitude for differing magnitudes of cohesive strength. In the Mohr-Coulomb regime the overburden stress increases the yield stress such that the relative crater depth decreases with increasing meteorite radius. In the von Mises regime the depth is independent of the cohesive strength and depends only on the von Mises limit. In the gravity regime, crater depth is independent of the material strength. For example, as is shown in Figure 9, in the case of the moon, for a von Mises limit of $Y_{3}=2.7 \times 10^{-3}$ Mbar the Mohr-Coulomb regime extends from meteorite radii

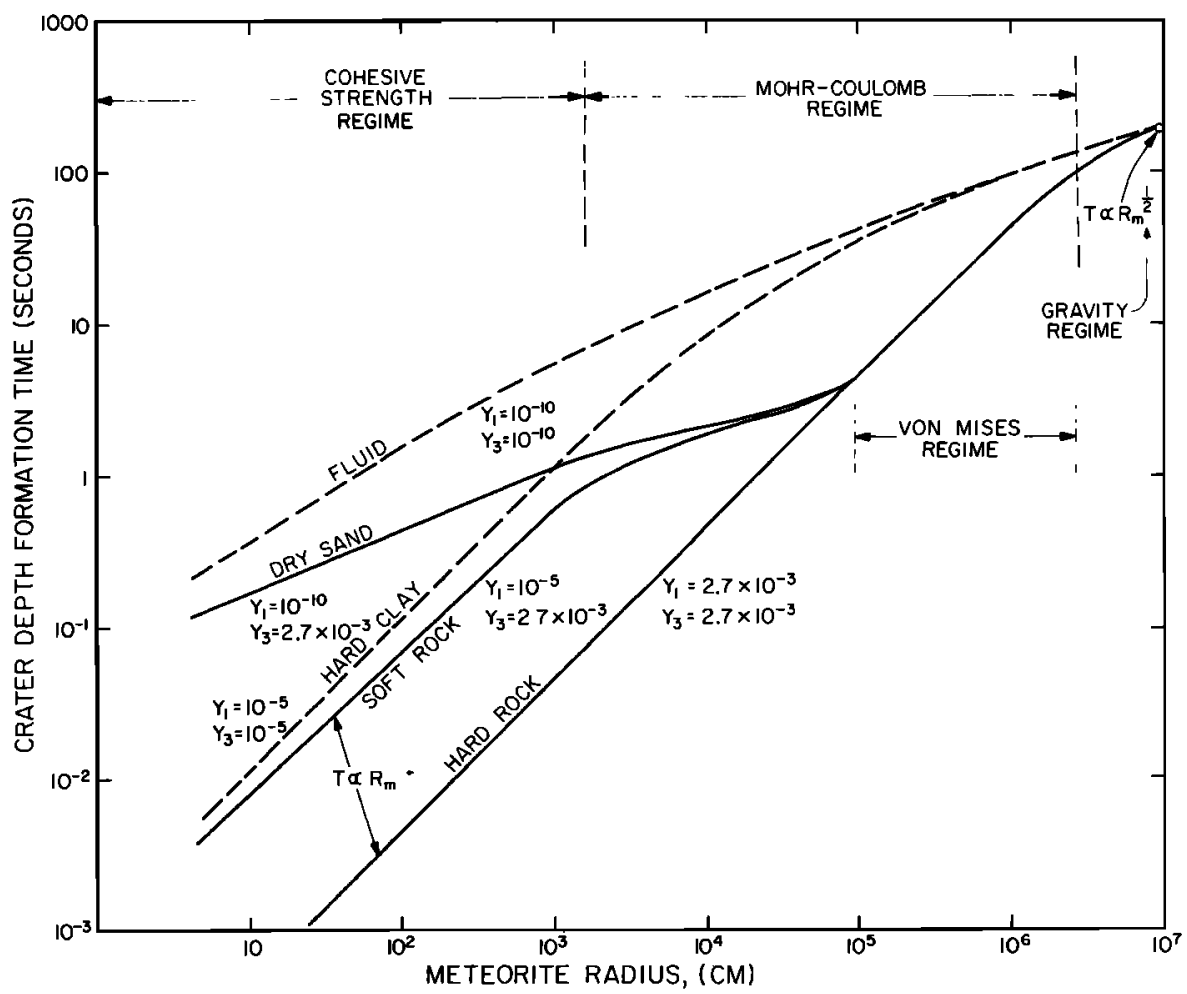

Fig. 8. Crater depth formation time for a $5-\mathrm{km} / \mathrm{s}$ impact on the moon versus anorthosite meteorite radius for various Mohr-Coulomb strength models. 
of $\sim 10^{2}$ to $10^{5} \mathrm{~cm}$, the von Mises regime extends from $\sim 10^{5}$ to $\sim 10^{7} \mathrm{~cm}$, and the gravity regime extends from $\sim 10^{7} \mathrm{~cm}$ to greater values.

A series of calculations were carried out using the shock degradation model specified by (76) with $\gamma=1$. In this model the peak shock wave pressure reduces the magnitude of the cohesive strength $Y_{1}$ and not the von Mises limit $Y_{3}$. The shock wave amplitudes used to degrade the value of $Y_{1}$ were obtained from the pressure decay calculations given by Ahrens and O'Keefe [1977]. The resulting relative crater depth as a function of meteorite radius for a $5-\mathrm{km} / \mathrm{s}$ impact is presented in Figure 10. The example chosen has an initially large cohesive strength which was equal to the von Mises limit, $Y_{1}=Y_{3}$ $=2.7 \times 10^{-3}$ Mbar. The strength degradation effect was expected to be most pronounced in this case. The passage of the shock wave reduced the cohesive strength to $10^{-10} \mathrm{Mbar}$ for shock pressures greater than $10^{-4} \mathrm{Mbar}$ in one case and $10^{-3}$ Mbar in another. As is demonstrated in Figure 10, in the cohesive regime (meteorite radius $<10^{5} \mathrm{~cm}$ ), shock degradation could be an important mechanism in determining crater depth. The crater depth more than doubles when rock is processed by a threshold shock pressure of $10^{-4} \mathrm{Mbar}$. The effect diminishes in the Mohr-Coulomb regime and vanishes in the von Mises and gravity regimes.

The effect of strength on the transient crater depth versus kinetic energy of impact $(E)$ is presented in Figure 11 on the basis of various strengths listed in Table 4 . We emphasize the word transient, because observationally, it has been found that the crater depths do not exceed $\sim 5 \mathrm{~km}$, regardless of diameter, on the moon, Mercury, and probably other planets. We believe that the limit observed in crater depth for larger craters results from later stages of crater development than those described here [e.g., Melosh, 1977; McKinnon, 1978]. Like the relationship of the transient crater growth times and crater depth versus meteorite radius, the impact energy can also be described as occurring within several regimes which are related to the assumed strength model. In the case of very weak materials (case 1 in Table 4) the crater depth is dominated by gravity, and the depth scales as $E^{1 / 4}$ [e.g., Chabai, 1965; Gault et al., 1975; Sauer, 1978]. In the case of very strong materials the crater depth scales as $E^{1 / 3}$ at impact energies of $\leq 10^{20}$ ergs, whereas when gravitational forces dominate, the crater depth scales as $E^{1 / 4}$ as energies approach $10^{30}$ ergs. In

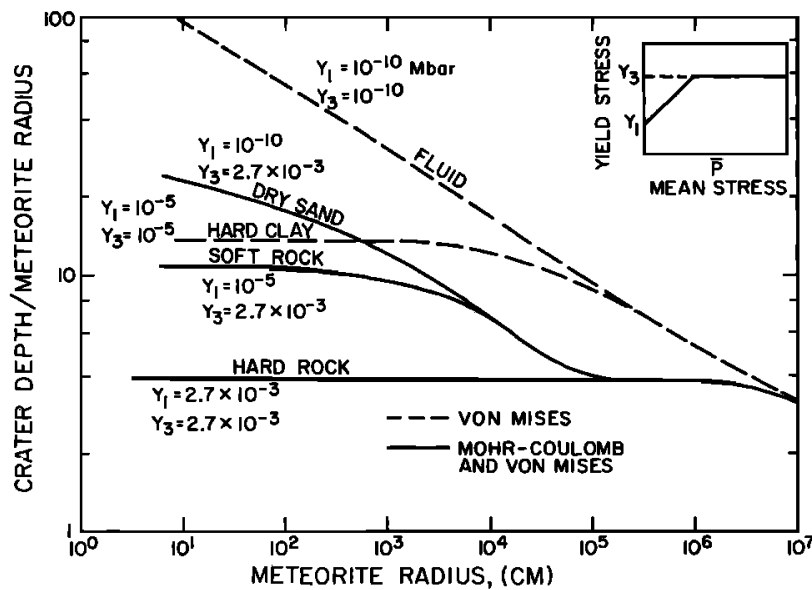

Fig. 9. Normalized crater depth versus anorthosite meteorite radius for a $5-\mathrm{km} / \mathrm{s}$ impact on the moon for different Mohr-Coulomb strength models.

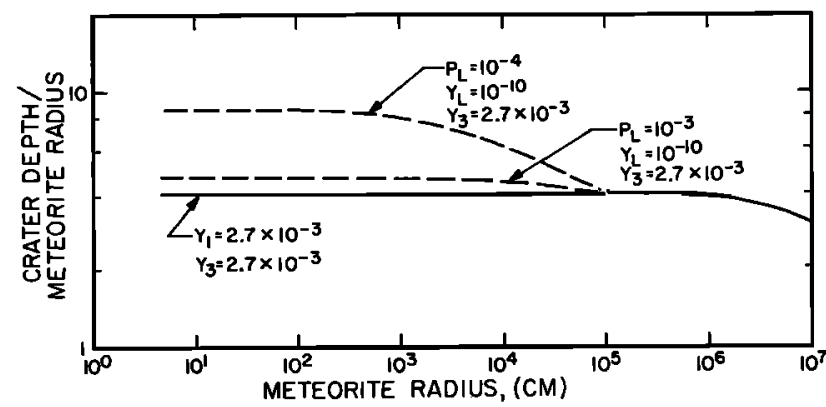

Fig. 10. Normalized crater depth versus anorthosite meteorite radius for a $5-\mathrm{km} / \mathrm{s}$ impact on the moon for different strength degradation models of the type shown in Figure $4 b$.

the case of Mohr-Coulomb materials (cases 2-5 in Table 4) and large von Mises limits (e.g., $Y_{3}=2.7 \times 10^{-3} \mathrm{Mbar}$ ) the depth scales as $E^{-1 / 34}$. This intermediate type of scaling was first proposed by Vaile [1961] on the basis of correlations of chemical and nuclear explosion data.

\section{COMPARISON OF INCOMPRESSIBLE MODEL With SAND CRATERING EXPERIMENTS}

The comparison of impact cratering experiments with detailed calculations has to date, surprisingly, only been carried out in the case of metals [Rosenblatt et al., 1970] and composite structures [Gehring, 1970].

Our objective was to find a set of well-controlled impact experiments in a medium of geologic interest and compare the incompressible flow model to those results so that we could have confidence in computing cratering over a broad range of conditions. The sand impact experiments of Gault and Wedekind [1977] provide an appropriate data base. The properties of the sand used in these experiments was measured and demonstrated a Mohr-Coulomb type behavior for which the yield stress in shear can be approximated by

$$
Y(\text { Mbar })=25 \times 10^{-16}+33 \times 10^{-14} \bar{P}
$$

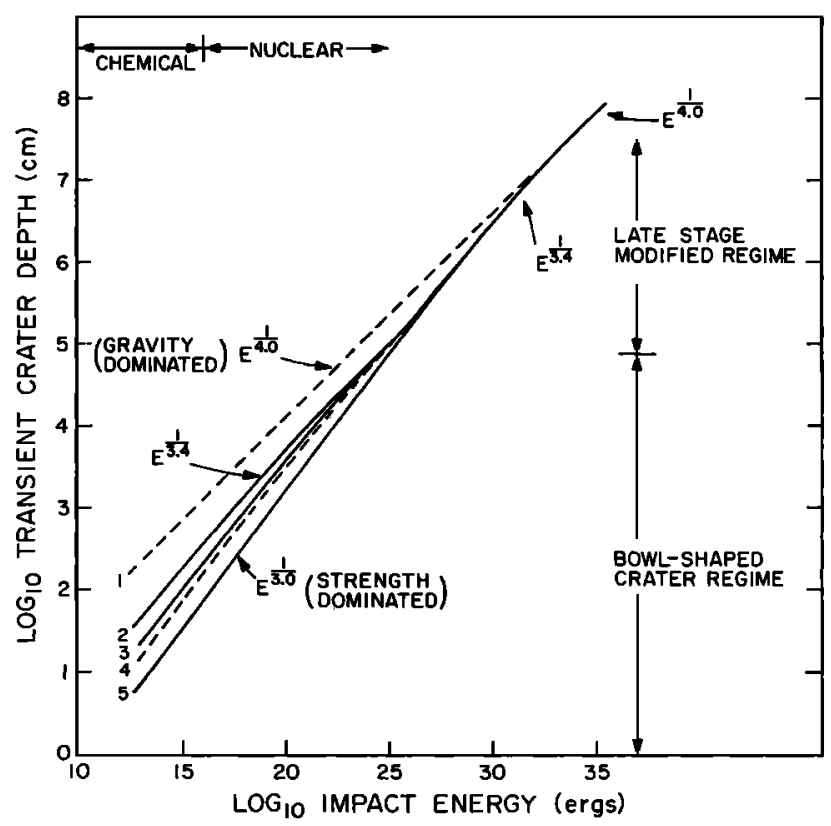

Fig. 11. $\log _{10}$ of transient crater depth versus anorthosite impactor energy for a $5-\mathrm{km} / \mathrm{s}$ impact on the moon for different strength models specified in Table 4. 
TABLE 5. Parameters of $z$ Model Used for Comparison With Experiments of Gault and Wedekind [1977]

\begin{tabular}{cl}
\hline Material Strength Parameter & Value \\
\hline$Y_{1}$, Mbar & $2.5 \times 10^{-16}$ \\
$Y_{2}, \tan \phi$ & 0.33 \\
$Y_{3}$, Mbar & $2.7 \times 10^{-3}$ \\
\hline
\end{tabular}

The material strength parameters were calculated from a graph of the data of Gault and Wedekind [1977]. The density is $1.65 \mathrm{~g} / \mathrm{cm}^{3}$, and the initial velocity is $0.075 \mathrm{~km} / \mathrm{s}$.

where $\bar{P}$ is the overburden stress, which is assumed to be equal to the average principal stress as used in (76). Gault and Wedekind's [1977] experiments were carried out on a size scale between that of cohesion- and that of gravity-dominated scaling and thus provide a stringent test of present modeling capability in the Mohr-Coulomb regime.

These experiments consisted of impacting aluminum projectiles into sand at velocities in the range up to $6 \mathrm{~km} / \mathrm{s}$. Measurements of the crater diameter as a function of time for various values of acceleration, simulating various surface gravities, were carried out. The depth to diameter ratios remained nearly constant over the range of test conditions, so that the scaling of characteristic crater diameter and depth formation times should be proportional (i.e., have the same scaling exponent).

We have made a comparison of the scaling of the crater formation time as a function of the size of impacting projectile and surface gravity. The objectives of these calculations were to show a correlation between theory and experiments of the scaling of crater depth with time and gravity. This scaling is insensitive to the exact magnitude of the initial velocity field. Because of this insensitivity we did not use the detailed compressible flow code to compute the initial flow field in sand but rather assumed the same flow field that we calculated previously [O'Keefe and Ahrens, 1977b], associated with impact of anorthosite upon an anorthosite half space at $5 \mathrm{~km} / \mathrm{s}$. This impact flow field was used to determine the initial transient cavity size $R_{0}, z$, and velocity magnitude $\alpha$ (e.g., (1) and (7)). A summary of the $z$ model parameters is given in Table 5 .

The crater depth formation time was computed by using the $z$ model as a function of crater depth ranging from $0.4 \mathrm{~cm}$ to 4 $\times 10^{3} \mathrm{~cm}$. The associated formation time varied from $3.5 \times$ $10^{-3}$ to $0.4 \mathrm{~s}$. Referring to Figure 12 , the slope of the calculated crater formation time or the scaling exponent is very close to the value found by Gault and Wedekind over the experimental range.

The crater depth formation time was also computed over a range of surface accelerations less than $\mathrm{lg}$. Referring to Figure 13 , the slope or scaling exponent in that case also agreed quite well with the value found by Gault and Wedekind $(t \propto$ $\left.g^{-0.618}\right)$. Note that for gravity-dominated craters the crater formation time scales as $g^{-0.625}$; thus the small shear strength of sand decreases the formation time in relation to strengthless gravity scaling. Finally, transient crater depth versus $\mathbf{g}$ is calculated over the range studied by Gault and Wedekind [1977] (except that they determined the diameter $D$ ). In Figure 14 we show that the calculated exponent of depth (or diameter) is again close to the value obtained by Gault and Wedekind [1977]. Both are less than the $D \propto g^{-0.25}$ expected for pure gravity scaling.

\section{Conclusions}

The effect of varying planetary crustal strength and surface gravity on the depth of impact craters was investigated by means of coupling the results of compressible flow finite difference calculations carried out to stress levels below the compressional dynamic yield point (Hugoniot elastic limit) with the incompressible fluid flow model of Maxwell [1973]. The incompressible formulation is applicable to the flow after the stress levels had decayed to $\cong 1.5 \mathrm{kbar}$, resulting from the impact of a $5-\mathrm{km} / \mathrm{s}$ anorthosite sphere on a half space of anorthosite in the gravity field of the moon. We found that, like the case of explosions, the radial particle velocity at a given time field can be represented as being proportional to $(1 / R)^{z}$, where $z \cong 2$ along the axis stream tube and $z \cong 3$ when averaged over a larger portion of the half space. We outlined the physical and mathematical framework of the Maxwell model. This model assumes that the kinetic energy within the incompressible flow generated by the passage of the shock wave in the crater excavation region is converted into elastic, plastic, and potential energy by doing work against the target's material strength and body forces (in this case, planetary gravity); thus the final crater depth and volume is determined from conservation laws. We developed expressions for the final crater depth in the strength-dominated case, for fixed gravity $g$, in terms of target density $\rho$ and yield strength $Y$, which is proportional to $\left[\rho u_{o r}{ }^{2} / Y z\right]^{1 /(z+1)}$, where $u_{o r}$ is the radial particle velocity on the surface of the early time cavity of radius $R_{0}$. In addition, we developed comparable expressions for the gravity-dominated case for a fixed material strength, in which the final crater depth is proportional to $\boldsymbol{R}_{0}\left[2(z+1) u_{a r}{ }^{2} / g\right]^{1 /(z+1)}$. When $Y$ is specified by a Mohr-Coulomb relation of the form $Y=Y_{1}+a \bar{P}$ up to a von Mises limit $Y_{3}$, analytical solutions were not found, and the effect of strength on the crater depth was computed numerically by using the lithostatic pressure in addition to the dynamic pressure resulting from the vorticity of the flow.

The effect of shock wave degradation of material strength on crater depth was examined. The cohesive strength $Y_{1}$ was modeled as decreasing with the exposure of rock to increased shock pressure and was found to have the effect of increasing strong rock crater excavation depths by as much as a factor of 2 for impactor radii of $₹ 10^{3} \mathrm{~cm}$.

The scaling of transient crater depth with impact energy was found in the case of the moon to be strength dominated for high-strength surfaces at impact energies $E \gtrless 10^{20} \mathrm{ergs}$ and hence scale as $E^{1 / 3}$, whereas for weak materials and for strong surfaces where the impact energy is $₹ 10^{30}$ ergs, the crater

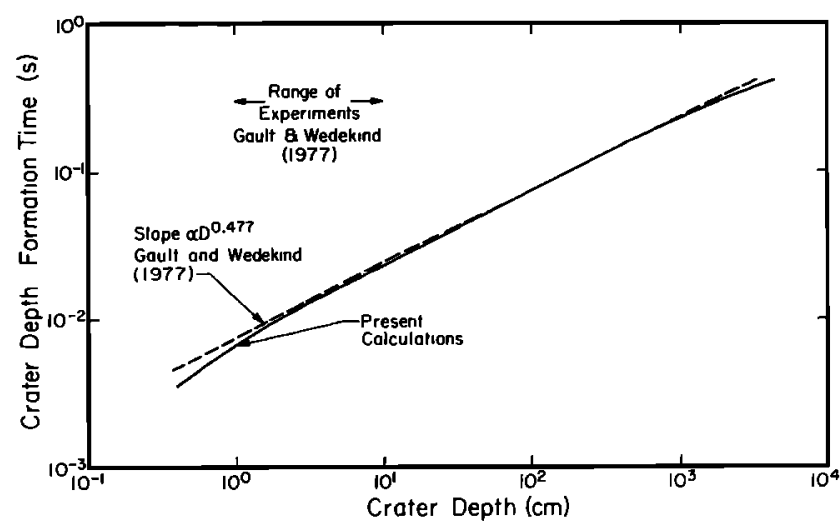

Fig. 12. Variation of crater depth formation time with crater depth using the present incompressible flow formulation with the assumption of a $5-\mathrm{km} / \mathrm{s}$ anorthosite-anorthosite velocity field. Shown also is the range of crater depths (diameters) observed in the sand experiments of Goult and Wedekind [1977] and the associated slope and scaling relationship. 
depths scale as $E^{1 / 4}$. However, for a broad range of observable crater sizes and expected strength parameters the depth scales as $\sim E^{1 / 3.4}$, which is consistent with chemical, nuclear, and impact crater statistics.

The transient crater depth and formation time was shown to vary greatly depending upon planetary strength. For impacting meteorite radii less than $10^{3} \mathrm{~cm}$ the crater formation time varied by about 5 orders of magnitude from $10^{-3}$ to $10 \mathrm{~s}$, and the crater depth to meteorite radius ratio varied from 4 to 32 ; this behavior is characteristic of what we call the cohesive strength regime. For meteorite radii greater than $10^{3} \mathrm{~cm}$ the crater depth decreased sharply with increasing meteorite radius for Mohr-Coulomb materials, and its rate was unchanged for von Mises materials. We denote this behavior with the terms Mohr-Coulomb and von Mises regimes. At sufficiently large meteorite radii (e.g., $\sim 10^{5}$ to $\sim 10^{6} \mathrm{~cm}$ ) the strength of Mohr-Coulomb materials is limited by the von Mises limit. Finally, for impactor radii of $\geq 10^{7} \mathrm{~cm}$ the crater depth and formation time are dominated by gravity.

The partitioning of energy in the axial stream tube was examined for impacts on the moon in which the surface strength was constant and high (2.7 kbar) versus one whose strength increased with confining pressure from $\sim 0.01$ to $2.7 \mathrm{kbar}$. For the former (von Mises type) material some $75 \%$ of the initial kinetic energy goes into reversible elastic strain energy, for meteorite radii less than $2 \times 10^{5} \mathrm{~cm}$, whereas for the latter (Mohr-Coulomb type) material, virtually all the impact energy is deposited as shock, or deformational, energy for impactors having a diameter less than $\sim 10^{4} \mathrm{~cm}$. Moreover, it was demonstrated that some $25 \%$ of the impact energy goes into work against the moon's gravity field for meteorite radii approaching $\sim 10^{7} \mathrm{~cm}$.

The experimental data of Gault and Wedekind [1977] for cratering in sand with energies of $6 \times 10^{7}$ to $10^{10}$ ergs under effective gravities of $0.07 \mathrm{~g}-1 \mathrm{~g}$ provide a test of both strength effects and gravity on the present incompressible flow calculations. The starting condition for the calculations was the scaled flow field for an anorthosite sphere impacting an anorthosite half space at $5 \mathrm{~km} / \mathrm{s}$. Using Gault and Wede-

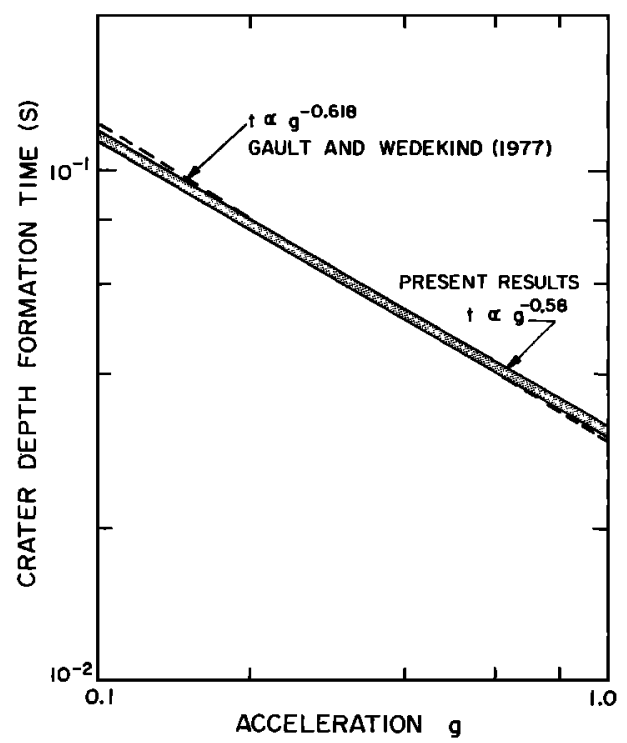

Fig. 13. Variation of crater depth formation time with gravity using the present incompressible flow formulation with the assumption of a $5-\mathrm{km} / \mathrm{s}$ anorthosite-anorthosite velocity field. Shown also is the slope and scaling relationship found by Gault and Wedekind [1977] in sand experiments.

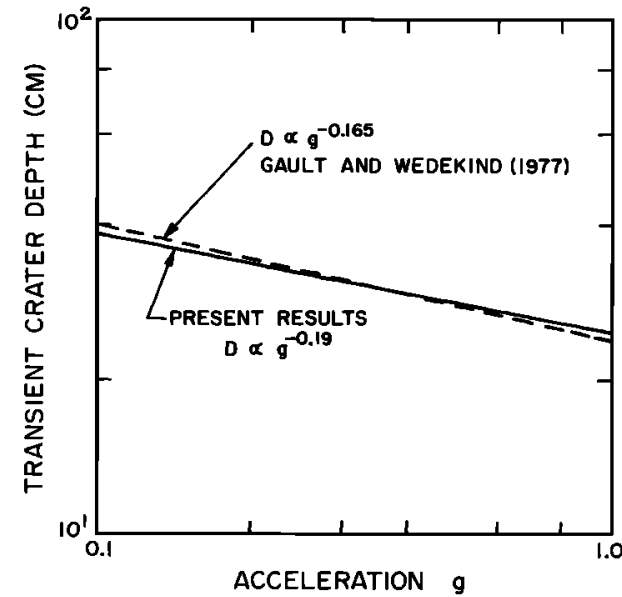

Fig. 14. Variation of transient crater depth $D$ with gravity using the present incompressible flow formulation with the assumption of a $5-\mathrm{km} / \mathrm{s}$ anorthosite-anorthosite velocity field. Shown also is the slope and scaling relationship found by Gault and Wedekind [1977] in sand experiments.

kind's reported shear yield strengths, crater formation times were found to vary with gravity as $g^{-0.58}$, whereas they observed a $g^{-0.618}$ dependence over a range from 0.03 to $0.12 \mathrm{~s}$. Similarly, a maximum crater depth dependence was calculated which varied as $\mathbf{g}^{-0.19}$, whereas the diameter dependence of Gault and Wedekind's experiments followed a $g^{-0.165}$ dependence. The comparison of the present incompressible flow calculation with experiments demonstrates the need for data describing the strength properties of planetary surfaces in order to relate crater size populations to impact history on planets with differing surface materials and gravities.

Acknowledgments. We are grateful for both the help and the access to unpublished materials so generously proffered by D. E. Maxwell of Science Applications, Incorporated. The final manuscript has benefited from the rigorous reviews of $R$. Schmidt of the Boeing Company and $H$. Moore of the USGS. Technical discussions with B. Minster and $\mathrm{J}$. Melosh and computational assistance by $M$. Lainhart are also appreciated. The research was supported under NASA grant NSG 7129. Contribution 3300, Division of Geological and Planetary Sciences, California Institute of Technology.

\section{REFERENCES}

Ahrens, T. J., and J. D. O'Keefe, Equations of state and impact-induced shock-wave attenuation on the moon, in Impact and Explosion Cratering, edited by D. J. Roddy, R. O. Pepin, and R. B. Merrill, pp. 639-656, Pergamon, New York, 1977.

Ahrens, T. J., J. D. O'Keefe, and R. V. Gibbons, Shock compression of a recrystallized anorthositic rock from Apollo 15, Geochim. Cosmochim. Acta, 3, 2575-2590, 1973.

Austin, M. G., J. M. Thomsen, and S. F. Ruhl, Calculational investigation of impact cratering dynamics: Material motions during the crater growth period (abstract), Lunar Planet. Sci., XI, 46-48, 1980.

Batchelor, G. K., An Introduction to Fluid Mechanics, 615 pp., Cambridge University Press, New York, 1967.

Bjork, R. L., Analysis of the formation of Meteor Crater, Arizona: A preliminary report, J. Geophys. Res., 66, 3379-3388, 1961.

Bjork, R. L., K. N. Kreyenhagen, and M. H. Wagner, Analytic study of impact effects as applies to the meteoroid hazard, NASA Contract. Rep., CR-757, 1967.

Bryan, J. B., D. E. Burton, M. E. Cunningham, and L. A. Lettis, Jr., A two-dimensional computer simulation of hypervelocity impact cratering: Some preliminary results for Meteor Crater, Arizona, Proc. Lunar Sci. Conf. 9th, 3931-3964, 1978.

Chabai, A. J., On scaling dimensions of craters produced by buried explosions, J. Geophys. Res., 70, 5075-5098, 1965

Charters, A. C., and J. L. Summers, Some comments on the phenom- 
ena of high speed impact, Rep. NOLTR 1238, pp. 200-201, U.S. Nav. Ordnance Lab., White Oak, Silver Spring, Md., 1959.

Dienes, J. K., and J. M. Walsh, Theory of impact: Some general principles of the method of Eulerian codes, in High Velocity Impact Phenomena, edited by R. Kinslow, pp. 45-156, Academic, New York, 1970.

Gaffiney, E. S., Effects of gravity on explosive-craters (abstract), Lunar Planet. Sci. Conf. 9th, 3831-3842, 1979.

Gault, D. E., Displaced mass, depth, diameter and effects of oblique trajectories for impact craters formed in dense crystalline rocks, Moon, 6, 32-44, 1973.

Gault, D. E., and J. A. Wedekind, Experimental hypervelocity impact in quartz sand, II, Effects of gravitational acceleration, Impact and Explosion Cratering, edited by D. J. Roddy, R. O. Pepin, and R. B. Merrill, pp. 1231-1234, Pergamon, New York, 1977.

Gault, D. E., J. E. Guest, J. B. Murray, D. Dzurisin, and M. C. Malin, Some comparisons of impact craters on Mercury and the moon, $J$. Geophys. Res., 80, 2444-2460, 1975.

Gehring, J. W., Jr., Theory of impact on correlation with experiment, in High Velocity Impact Phenomena, edited by R. Kinslow, pp. 105156, Academic, New York, 1970.

Holsapple, K. A., and R. M. Schmidt, A material-strength model for apparent crater volume, Proc. Lunar Planet. Sci. Conf. 10th, 2757$2777,1979$.

Johnson, S. W., J. A. Smith, E. G. Franklin, L. K. Morashi, and D. J. Teal, Gravity and atmospheric pressure effects on crater formation in sand, J. Geophys. Res., 74, 4838-4850, 1969.

Knowles, C. P., and H. L. Brode, The theory of cratering phenomena: An overview, in Impact and Explosion Cratering, edited by D. J. Roddy, R. O. Pepin, and R. B. Merrill, pp. 869-895, Pergamon, New York, 1977.

Maxwell, D. E., Cratering flow and crater prediction methods, Rep. TCAM 73-17, 50 pp., Phys. Int., San Leandro, Calif., 1973.

Maxwell, D. E., Simple $Z$ model of cratering, ejection, and the overturned flap, in Impact and Explosion Cratering, edited by D. J. Roddy, R. O. Pepin, and R. B. Merrill, pp. 1003-1008, Pergamon, New York, 1977.

Maxwell, D. E., and R. Seifert, Modeling of cratering, close-in displacements and ejecta, Rep. DNA 3628F, p. 111, Phys. Int., San Leandro, Calif., 1976.

McKinnon, W. B., An investigation into the role of plastic failure in crater modification, Proc. Lunar Planet. Sci. Conf. 9th, 3965-3973, 1978.

Melosh, H. J., Crater modification by gravity: A mechanical analysis of slumping, in Impact and Explosion Cratering, edited by D. J. Roddy, R. O. Pepin, and R. B. Merrill, pp. 1245-1260, Pergamon, New York, 1977.

Moore, H. J., D. E. Gault, and E. D. Heitowit, Change of effective target strength with increasing size of craters for hypervelocity im pact craters, Proc. Hypervelocity Impact Symp. 7th, 4, 35-45, 1964.

Neukum, G., Lunar cratering, Philos. Trans. R. Soc. London, Ser. A, 285, 267-272, 1977.

Neukum, G., and D. V. Wise, Mars: A standard crater curve and possible new time scale, Science, 194, 1381-1387, 1976.

Oberbeck, V. R., Laboratory simulation of impact cratering with high explosives, J. Geophys. Res., 76, 5732-5749, 1971.

O'Keefe, J. D., and T. J. Ahrens, Shock effects from a large impact on the moon, Proc. Lunar Sci. Conf. 6th, 2831-2844, 1975.

O'Keefe, J. D., and T. J. Ahrens, Impact ejecta on the moon, Proc. Lunar Sci. Conf. 7th, 3007-3025, 1976.

O'Keefe, J. D., and T. J. Ahrens, Meteorite impact ejecta: Dependence of mass and energy lost on planetary escape velocity, Science, 198, 1249-1251, 1977a.

O'Keefe, J. D., and T. J. Ahrens, Impact-induced energy partitioning, melting and vaporization on terrestrial planets, Proc. Lunar Sci. Conf. 8th, 3357-3374, $1977 b$.

O'Keefe, J. D., and T. J. Ahrens, Impact flows and crater scaling on the moon, Phys. Earth Planet. Inter., 16, 341-351, 1978.

Orphal, D. L., Calculations of explosion cratering, II, Cratering mechanics and phenomenology, in Impact and Explosion Cratering, edited by D. J. Roddy, R. O. Pepin, and R. B. Merrill, pp. 907-917, Pergamon, New York, 1977.

Rosenblatt, M., J. J. Piechocki, and K. N. Kreyenhagen, Cratering and surface waves caused by detonation of a small explosive charge in aluminum, DASA Rep. 2495, Shock Hydrodynamics, Sherman Oaks, Calif., 1970.

Sauer, F. M., The influence of target material strength on the scaling of cratering and ejecta data, Lunar Planet. Sci., IX, 993-995, 1978.

Schmidt, R. M., and K. A. Holsapple, Theory and experiments on centrifuge cratering, J. Geophys. Res., 85(B1), 235-252, 1980.

Swift, R. P., Material strength degradation effect on cratering dynamics, in Impact and Explosion Cratering, edited by D. J. Roddy, R. $O$. Pepin, and R. B. Merrill, pp. 1025-1042, Pergamon, New York, 1977.

Vaile, R. B., Jr., Pacific craters and scaling laws, J. Geophys. Res., 66 3413-3438, 1961.

Viktorov, V. V., and R. D. Stepenov, Modeling of the action of an explosion with concentrated charge in homogeneous ground (in Russian), Inzh. Sb., 28, 87-96, 1960. (English translation, Rep. SCL-T 392, Sandia Lab., Albuquerque, N. M., 1961.)

Whitaker, E. A., Artificial lunar impact craters: Four new identifications, NASA Spec. Publ., SP-315, 29-39-29-45, 1972.

(Received September 1, 1979;

revised July 1,1980 ;

accepted July 21,1980 .) 